\title{
Análise espacial da compactação do solo causada pelo sistema de colheita de madeira de árvores inteiras ${ }^{1}$
}

\author{
Carla Krulikowski Rodrigues ${ }^{2}$,Eduardo da Silva Lopes ${ }^{2 *}$
}

$10.1590 / 0034-737 X 201865030002$

\section{RESUMO}

A análise espacial da compactação do solo causada pelo sistema de colheita da madeira pode auxiliar no planejamento das operações florestais. Objetivou-se, com este trabalho, identificar e mapear os níveis de compactação do solo, causados pelas máquinas, ao longo das trilhas de extração, pelo sistema de colheita de madeira de árvores inteiras. Foi estudado o sistema de colheita da madeira composto por feller buncher, skidder e harvester, sendo instaladas quatro malhas amostrais nas diferentes distâncias de extração da madeira (0 a 50; 51 a 100; 101 a 150 e 151 a $200 \mathrm{~m}$ ) e avaliada a compactação do solo em quatro camadas de profundidade ( 0 a 10; 11 a 20; 21 a 40 e 41 a $60 \mathrm{~cm})$, a fim de verificar o efeito da variação do tráfego do skidder, ao longo do talhão, por estudo das resistências do solo à penetração, com penetrômetro digital. A partir da técnica de geoestatística foram elaborados mapas temáticos e quantificadas as áreas com os diferentes níveis de compactação. Os resultados permitiram localizar as regiões ao longo do talhão com diferentes intensidades de compactação, sendo que a região próxima à margem do talhão apresentou os maiores níveis de compactação, com resistência à penetração acima de 2 Mpa e comprometendo em torno de $20 \%$ da área total, na primeira classe de distância. As demais classes de distância de extração não atingiram níveis significativos de compactação, mostrando que o aumento da intensidade de tráfego não influenciou as propriedades físicas do solo. A técnica de geoestatística mostrou-se viável para a determinação da compactação do solo nas diferentes regiões do talhão, podendo favorecer o planejamento das operações de preparo de solo.

Palavras-chave: física do solo; geoestatística; penetrometria; colheita florestal.

\section{ABSTRACT}

\section{Spatial analysis of soil compaction caused by the full tree wood harvesting system}

The spatial analysis of soil compaction caused by the wood harvesting system can assist in forest operation planning. The aim of this work was to identify and map the levels of soil compaction along the traces of wood extraction caused by the machines in the harvesting full tree system. We studied the wood harvesting system composed of feller buncher, skidder, and harvester, with sample meshes installed at different wood extraction distances ( 0 to 50; 51 to 100 ; 101 to 150 , and 151 to $200 \mathrm{~m}$ ) and soil compaction in four depths ( 0 to $10 ; 11$ to $20 ; 21$ to 40 , and 41 to $60 \mathrm{~cm}$ ) to verify the effect of the variation of the skidder traffic along the field through the penetration resistance of the soil with the use of a digital penetrometer. Through the geostatistics technique, thematic maps were elaborated and the areas with the different levels of compaction were quantified. The results allowed to locate the regions along the plot with different intensities of compaction, in which the region next the margin of the plot presented greater soil compaction with a resistance to penetration above $2 \mathrm{MPa}$, compromising approximately $20 \%$ of the total area of the first-class distance. The other classes of extraction distance did not reach this level of compaction, showing that the increase of traffic intensity did not influence the soil physical properties. The geostatistical technique proved to be feasible in the determination of soil compaction in the different areas of the field and may favor the planning of soil preparation operations.

Keywords: soil physics; geostatistics; penetrometer; forest harvesting.

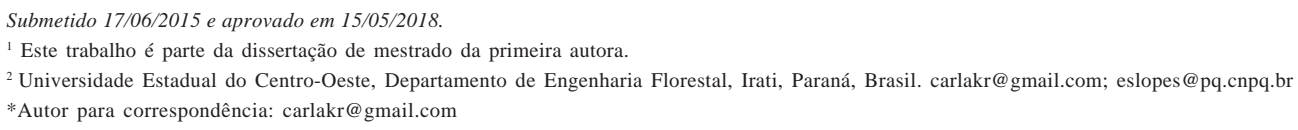




\section{INTRODUÇÃO}

As novas tecnologias para aumentar a produtividade e atender a demanda mundial por produtos florestais, mesmo que associadas às técnicas de conservação, têm acarretado diversas formas de degradação dos solos, sendo a compactação e a dimensão da área afetada por fatores de grande relevância, podendo alterar as propriedades físicas, químicas e biológicas do solo e, consequentemente, o potencial produtivo dos povoamentos florestais (Cambi et al., 2017; Epron et al., 2016).

A compactação é definida como a ação mecânica que impõe ao solo a redução de seu índice de porosidade. Trata-se da aproximação das partículas, com redução do volume por elas ocupado, resultando em tensões aplicadas e causando incremento da densidade e redução do espaço poroso, da infiltração e do movimento interno de água e em maior resistência mecânica ao crescimento das raízes (Reichert et al., 2010).

A compactação do solo em áreas florestais vem sendo amplamente estudada, podendo ser afetada por fatores como teor de matéria orgânica, textura, umidade, resíduos sobre o terreno, modelos de máquinas e tipos de rodados, tamanho e pressão dos pneus, intensidade de tráfego das máquinas, etc. (Reichert et al., 2007; Lopes et al., 2011).

Aspecto importante, a ser considerado no planejamento das operações florestais, consiste na seleção do sistema de colheita da madeira, viável do ponto de vista técnico, econômico e ambiental, pois, dependendo do sistema utilizado, a compactação do solo pelas máquinas poderá afetar o ecossistema, de forma aleatória ou sistemática, na área do povoamento florestal.

Segundo Machado (2014), predominam no Brasil dois sistemas de colheita da madeira: o de toras curtas e o de árvores inteiras. O primeiro é caracterizado principalmente pelo uso do harvester e do forwarder; nele as árvores são derrubadas e processadas no interior do talhão, sendo a madeira, em seguida, extraída para a margem do talhão, na forma de toras, com concentração do tráfego das máquinas em carreadores pré-designados no interior do talhão. Por outro lado, o sistema de árvores inteiras é caracterizado, predominantemente, pelo uso do feller buncher, do skidder e do harvester: as árvores são derrubadas e, em seguida, arrastadas até a margem dos talhões onde é realizado o processamento final da madeira, com tráfego aleatório das máquinas no interior do talhão.

A compactação do solo na colheita de madeira tem ocorrido com maior frequência em função do aumento do tráfego de máquinas de grande porte. Seixas \& Souza (2007) afirmam que existe a necessidade de estabelecer maiores restrições ao tráfego no interior do talhão, diminuindo-se, assim, a extensão dos prováveis efeitos sobre o povoamento florestal. Silva et al. (2004) afirmam que a compactação ocorre com maior intensidade nas áreas próximas às margens do talhão, situação comum no sistema de colheita de árvores inteiras.

Dias Junior et al. (2003), ao avaliarem dois sistemas de colheita da madeira em um Latossolo Vermelho-Amarelo, afirmaram que a compactação apresentou distribuição aleatória no sistema de árvores inteiras, abrangendo maior área do talhão, em comparação com a verificada no sistema de toras curtas, cujos impactos foram sistemáticos, com localização definida e em menor área do talhão. Por fim, Lima et al. (2008) constataram, na seção transversal à trilha de tráfego do feller buncher e do skidder, por meio de técnicas de geoestatística, que a compactação ocorreu após as passadas das máquinas, ficando restrita nas camadas superficiais do solo.

Diante disso, objetivou-se, com este trabalho, realizar avaliação espacial da compactação do solo, causada pelo tráfego das máquinas de colheita da madeira no sistema de árvores inteiras, gerando informações que possam contribuir para o planejamento e a sustentabilidade da produção florestal.

\section{MATERIAL E MÉTODOS}

O estudo foi realizado nas áreas de colheita da madeira de uma empresa localizada na Região dos Campos Gerais, Estado do Paraná, Brasil, entre as coordenadas geográficas $24^{\circ} 19^{\prime} 26^{\prime \prime} \mathrm{Se} 50^{\circ} 36^{\prime} 58^{\prime \prime} \mathrm{O}$, com altitude média de $760 \mathrm{~m}$.

O clima predominante da região de estudo, de acordo com o sistema de classificação climática de Köppen, está situado entre a região $\mathrm{Cfa}$ e $\mathrm{Cfb}$, com predomínio do Cfa, apresentando temperatura média no mês mais frio inferior a $18^{\circ} \mathrm{C}$ (mesotérmico) e temperatura média no mês mais quente, acima de $22^{\circ} \mathrm{C}$. A precipitação média anual no ano do estudo foi de $1.351 \mathrm{~mm}$ (AguasParaná, 2018).

O solo da área de estudo foi classificado como Latossolo Vermelho franco-argilo-arenoso, sendo a análise granulométrica realizada pelo método da pipeta, proposto pela Embrapa (1997), juntamente com a determinação da umidade gravimétrica. $\mathrm{O}$ teor de carbono orgânico foi obtido pelo método Walkley-Black, proposto por Pavan et al. (1992).

O relevo da área do estudo foi classificado como de plano a suavemente ondulado, com declividade média de $6 \%$. O regime de manejo adotado pela empresa foi o corte raso, sendo a madeira destinada à produção de celulose. As características do povoamento florestal estudado estão descritas na Tabela 1.

Foi estudado o sistema de colheita de árvores inteiras, composto por feller buncher, skidder e harvester, máquinas responsáveis pelas operações de derrubada e empilhamento das árvores, arraste dos feixes de árvores e 
processamento da madeira, respectivamente. O feller buncher possuía motor com potência de $300 \mathrm{HP}$, peso operacional de $31,3 \mathrm{Mg}$, rodados de esteiras, cabeçote com peso operacional de 2,63 Mg e diâmetro máximo de corte de 58,5 cm; o skidder possuía motor com potência de $250 \mathrm{HP}$, peso operacional de $21,4 \mathrm{Mg}$, rodados de pneus recobertos com semiesteiras com tração 6x6 e garra com área útil de 1,95 m²; e o harvester possuía motor com potência de $294 \mathrm{HP}$, peso operacional de $28,7 \mathrm{Mg}$ e rodados de esteiras.

O estudo foi realizado logo após finalizadas as operações de corte e extração da madeira, sendo a compactação determinada por meio da resistência do solo à penetração (RP). Foi utilizado um penetrógrafo eletrônico digital da marca Eijkelkamp, modelo 06.15.SA, equipado com ponta tipo cone com ângulo de vértice de $60^{\circ}$ e base de $1 \mathrm{~cm}^{2}$, com a velocidade de penetração máxima de $2 \mathrm{~cm} \mathrm{~s}^{-1}$.

A avaliação da compactação causada pelas diferentes intensidades de tráfego das máquinas, ao longo das trilhas de extração, foi realizada em dois ramais com comprimento de 200 m, sendo esta a distância máxima de extração da área, no momento de realização do estudo. Os ramais de tráfego das máquinas foram subdivididos em quatro classes de distâncias: 0 a 50, 51 a 100, 101 a 150 e 151 a $200 \mathrm{~m}$, correspondendo a uma passada do feller buncher e 14, 10, 6 e 2 passadas do skidder, respectivamente, considerando-se suas viagens vazias e carregadas (Figura 1).

Foram estabelecidas, em cada classe de distância de extração, malhas amostrais retangulares (XY), com 150 pontos de RP, sendo 15 pontos na abscissa $(\mathrm{X})$, nas distâncias de 0,$0 ; 2,0 ; 4,0 ; 4,5 ; 5,0 ; 5,5 ; 7,5 ; 9,0 ; 10,5 ; 12,5$; 13,$0 ; 13,5 ; 14,0 ; 16,0 ; 18,0 \mathrm{~m}$, abrangendo dois ramais de extração, e dez pontos na ordenada (Y), equidistantes de um metro entre si, nas profundidades de 0 a 10; 11 a 20; 21 a 40 e 41 a $60 \mathrm{~cm}$.

Foi utilizado o programa GS+, cujos dados de RP foram submetidos à análise estatística descritiva, de modo a verificar a posição e a dispersão dos dados. Em seguida, determinaram-se os seguintes parâmetros estatísticos: Valores mínimos da RP(MPa), valores médios (MPa), mediana (MPa), valores máximos (MPa), coeficiente de vari-

Tabela 1: Características do povoamento florestal

\begin{tabular}{lc}
\hline Características do povoamento & Informações \\
\hline Espécie & Eucalyptus grandis \\
Idade de corte (anos) & 7 \\
Espaçamento $(\mathrm{m})$ & $2,5 \times 2,5$ \\
Área basal $\left(\mathrm{m}^{2} \mathrm{ha}^{-1}\right)$ & $38,0 \pm 3,34$ \\
DAP médio $(\mathrm{cm})$ & $19,2 \pm 1,94$ \\
Altura média $(\mathrm{m})$ & $27,6 \pm 2,20$ \\
Volume médio individual $\left(\mathrm{m}^{3}\right)$ & $0,36 \pm 0,04$ \\
Volume médio $\left(\mathrm{m}^{3} \mathrm{ha}^{-1}\right)$ & $430 \pm 59,40$ \\
\hline
\end{tabular}

\pm : desvio padrão. ação (CV, em \%), assimetria (Ass), curtose (Curt), nas diferentes profundidades $(\mathrm{cm})$ e classes de distância $(\mathrm{m})$.

Com relação aos valores de CV obtidos, a dispersão dos dados foi classificada em: baixa, quando os valores são menores que $12 \%$; média, com valores entre 12 e $60 \%$ e alta, com valores acima de $60 \%$.

Os ajustes dos modelos linear, esférico, gaussiano e exponencial foram realizados com base no melhor coeficiente de determinação $\left(\mathrm{R}^{2}\right)$. A partir do ajuste de um modelo matemático dos dados, foram definidos os seguintes parâmetros: efeito pepita $\left(\mathrm{C}_{0}\right)$, contribuição $\left(\mathrm{C}_{1}\right)$, patamar $\left(\mathrm{C}_{0}+\mathrm{C}_{1}\right)$ e alcance (a). O índice de dependência espacial (IDE) foi calculado por meio da equação: $\mathrm{IDE}=\left[\mathrm{C}_{0} /\left(\mathrm{C}_{0}+\right.\right.$ $\left.\left.\mathrm{C}_{1}\right)\right] \times 100$. Com base nos IDE, classificou-se o grau de dependência espacial (GDE) como: forte, para IDE > 25\%; moderado, para IDE entre 25 e $75 \%$; fraco, para IDE $>75 \%$ (Cambardella et al., 1994).

Em seguida, foram elaborados mapas temáticos horizontais da RP, nas diferentes classes de distâncias de extração e profundidades estudadas, com o programa Surfer 8.0, a partir de valores estimados por krigagem e dos parâmetros de semivariogramas ajustados. Por fim, para a quantificação dos percentuais de área, referentes a cada classe da resistência do solo à penetração, foi utilizado o programa Idrisi Taiga, de modo a identificar as zonas

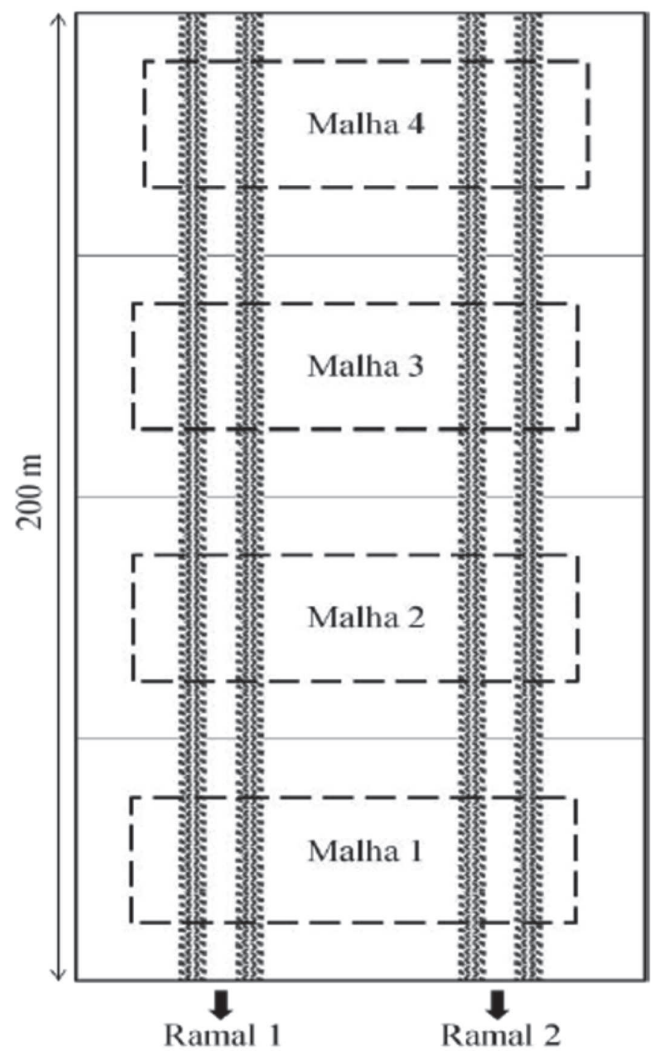

Figura 1: Croqui da alocação das malhas ao longo da trilha de extração de madeira. 
homogêneas e localizar e mensurar as áreas afetadas pelo tráfego das máquinas de colheita da madeira.

\section{RESULTADOS E DISCUSSÃO}

Os teores de areia grossa, areia fina, silte e argila, o teor médio de carbono orgânico, na camada de 0 a $60 \mathrm{~cm}$, e a umidade gravimétrica média, no momento de realização do estudo, estão apresentados na Tabela 2.

Na Tabela 3, estão apresentados os valores da RP, cujas médias variaram de $0,778 \mathrm{MPa}$ a $1,733 \mathrm{MPa}$. Porém, ao se observar os valores máximos apresentados, verifica-se que a classe de distância de 0 a 50 m apresentou valores acima de $2 \mathrm{Mpa}$, em todas as camadas de profundidade do solo avaliadas, enquanto, nas demais classes de distância de extração, os valores máximos acima de $2 \mathrm{MPa}$ estavam nas camadas de profundidade inferiores. De acordo com Lapen et al. (2004), uma RP de 2 MPa é considerada como valor médio crítico para o desenvolvimento das plantas, enquanto Zou et al. (2000) o consideram como sendo de $3 \mathrm{MPa}$, classificado como limite restritivo para o desenvolvimento de espécies florestais.
Nas profundidades estudadas, a RP apresentou CV de 15,54 a 37,65\%, podendo ser classificada como média. Em relação às medidas de posição central, observou-se que os valores das médias e das medianas foram similares, indicando que os dados apresentaram distribuição de frequência normal. A normalidade dos dados pode ser comprovada pelos valores de assimetria e da curtose próximos à zero. E mesmo não sendo isso uma exigência, a normalidade dos dados possibilitou melhor predição dos valores não amostrados por meio da geoestatística (Webster \& Oliver, 2007).

Os modelos ajustados aos semivariogramas (linear, esférico, gaussiano e exponencial), selecionados como os mais representativos estão descritos na Tabela 4. A maioria das camadas do solo apresentou ajuste ao modelo linear, correspondendo a maiores dispersão e variabilidade dos dados. Porém, o alcance tem grande importância para a interpretação dos semivariogramas, pois representou a distância em que os pontos amostrais se correlacionam entre si, podendo-se, então, afirmar que os pontos amostrais localizados em uma área onde a distância entre eles seja o alcance são mais semelhantes entre

Tabela 2: Granulometria média, carbono orgânico e umidade gravimétrica do solo, nas profundidades avaliadas

\begin{tabular}{|c|c|c|c|c|c|c|c|}
\hline Prof. & Areia grossa & Areia fina & Areia total & Silte & Argila & $\mathrm{CO}$ & $\mathbf{U g}$ \\
\hline (cm) & & & $\left(\mathrm{g} \mathrm{kg}^{-1}\right)$ & & & $\left(\mathrm{g} \mathrm{dm}^{-3}\right)$ & $\left(\mathrm{kg} \mathrm{kg}^{-1}\right)$ \\
\hline 0 a 10 & 386 & 245 & 631 & 71 & 298 & 9,72 & 0,28 \\
\hline 11 a 21 & 391 & 233 & 624 & 71 & 305 & 7,39 & 0,25 \\
\hline 21 a 41 & 401 & 230 & 631 & 73 & 296 & 8,94 & 0,27 \\
\hline 41 a 60 & 403 & 244 & 648 & 68 & 284 & 8,94 & 0,26 \\
\hline Média & 395 & 238 & 633 & 71 & 296 & 8,75 & 0,27 \\
\hline
\end{tabular}

Tabela 3: Valores mínimos de RP (MPa), média (MPa), mediana (MPa), máximo (MPa), coeficiente de variação (\%), assimetria (Ass), curtose (Curt), em diferentes profundidades do solo $(\mathrm{cm})$ e classes de distância $(\mathrm{m})$

\begin{tabular}{|c|c|c|c|c|c|c|c|c|}
\hline Distância (m) & Prof. (cm) & Mín. & média & Mediana & Máx. & $\mathrm{CV}$ & Ass & Curt \\
\hline \multirow{4}{*}{0 a 50} & 0 a 10 & 0,390 & 1,211 & 1,233 & 2,490 & 33,03 & 0,30 & 0,43 \\
\hline & 11 a 20 & 0,460 & 1,485 & 1,520 & 2,770 & 32,73 & 0,37 & 0,17 \\
\hline & 21 a 40 & 0,510 & 1,583 & 1,589 & 2,830 & 31,02 & 0,35 & $(-0,10)$ \\
\hline & 41 a 60 & 0,470 & 1,733 & 1,747 & 2,930 & 29,14 & $(-0,47)$ & $(-0,02)$ \\
\hline \multirow{4}{*}{51 a 100} & 0 a 10 & 0,210 & 0,877 & 0,890 & 1,480 & 30,22 & 0,14 & $(-0,56)$ \\
\hline & 11 a 20 & 0,210 & 1,066 & 1,021 & 2,620 & 33,96 & 1,18 & 3,70 \\
\hline & 21 a 40 & 0,370 & 1,259 & 1,297 & 1,890 & 28,83 & $(-0,58)$ & $(-0,16)$ \\
\hline & 41 a 60 & 0,210 & 1,416 & 1,461 & 2,040 & 23,59 & $(-1,40)$ & 3,55 \\
\hline \multirow{4}{*}{101 a 150} & 0 a 10 & 0,290 & 0,778 & 0,796 & 1,470 & 32,52 & 0,23 & $(-0,07)$ \\
\hline & 11 a 20 & 0,300 & 1,025 & 1,049 & 1,810 & 23,51 & 0,01 & 1,31 \\
\hline & 21 a 40 & 0,760 & 1,390 & 1,395 & 2,160 & 20,50 & 0,11 & $(-0,25)$ \\
\hline & 41 a 60 & 0,680 & 1,634 & 1,644 & 2,150 & 15,54 & $(-0,78)$ & 1,89 \\
\hline \multirow{4}{*}{151 a 200} & 0 a 10 & 0,260 & 0,911 & 0,851 & 1,710 & 37,65 & 0,56 & $(-0,11)$ \\
\hline & 11 a 20 & 0,460 & 1,113 & 1,065 & 1,740 & 26,86 & 0,51 & $(-0,37)$ \\
\hline & 21 a 40 & 0,440 & 1,329 & 1,349 & 1,830 & 23,25 & $(-0,39)$ & $(-0,26)$ \\
\hline & 41 a 60 & 0,210 & 1,469 & 1,550 & 2,120 & 25,80 & $(-1,15)$ & 1,57 \\
\hline
\end{tabular}

Rev. Ceres, Viçosa, v. 65, n.3, p. 227-233, mai/jun, 2018 
si, quando se compara com os pontos separados por distâncias maiores. Portanto, pode-se observar que o alcance, que variou de 1,20 a 15,40 m, estava acima da distância entre os pontos de amostragem.

A maioria dos modelos apresentou graus de dependência espacial do patamar moderados (25 a 75\%), em todas as profundidades estudadas, conforme classificação adaptada de Cambardella et al. (1994).

Na Figura 2, está ilustrado o mapeamento das resistências à penetração, obtidas nas diferentes classes de distância de extração e profundidades do solo, enquanto, na Tabela 5, está apresentada a quantificação percentual da área compactada em cada classe de RP do solo. Como se pode observar, na primeira classe de distância ( 0 a 50 m), a área avaliada apresentou aproximadamente $20,0 \%$ com elevada compactação, ou seja, próxima de 2 Mpa, enquanto, nas demais classes de distâncias, a compactação do solo não atingiu níveis significativos e prejudiciais ao desenvolvimento das plantas.

A profundidade de 21 a $40 \mathrm{~cm}$ não apresentou níveis de compactação considerados críticos ao desenvolvimento das plantas, porém é possível notar que, na primeira classe de distância ( 0 a 50 m) observou-se maior heterogeneidade dos valores de resistência à compactação, causada pela maior intensidade de tráfego do skidder neste local. Já a profundidade de 41 a $60 \mathrm{~cm}$ apresentou maior compactação na classe de distância de 0 a 50 m, com aproximadamente 25,3\% da área total e com valores acima de $2 \mathrm{Mpa}$, nível crítico para o desenvolvimento das plantas.

Portanto, foi possível verificar neste sistema de colheita, pouca visibilidade dos ramais trafegados pelas máquinas ao longo do talhão, porém a compactação apresentou maior ocorrência na camada de maior profundidade. Já nas camadas superficiais predominou uma intensidade de compactação de 1,20 a 1,49 MPa, enquanto, nas camadas mais profundas, obtiveram-se valores de 1,50 a 1,79 Mpa, devendo-se ressaltar que o aumento da resistência do solo à penetração na maior profundidade é considerado como característica de solos com altos teores de areia.

Em relação à dimensão da área compactada, os resultados entram em acordo com os de Seixas et al. (2003), que afirmam que o sistema de colheita de árvores inteiras tem a característica de não concentrar o tráfego das máquinas apenas nos ramais formados pelo feller-buncher, sendo que o skidder, ao realizar o arraste dos feixes de árvores, foi capaz de alterar em $30 \%$ a área plantada, com $85 \%$ da área total do talhão florestal apresentando algum tipo de distúrbio do solo.

O uso dos dados de penetrometria mostrou-se promissor para a determinação da espessura e da profundidade da camada compactada do solo. Além disso, dimensionar classes homogêneas de valores de resistência do solo à penetração é um importante avanço na evolução do manejo do solo, do uniforme para o localizado.

Portanto, pode-se dizer que cada classe de compactação do solo deverá receber um manejo diferenciado, em que, segundo Taylor et al. (2007), os custos para realização do dimensionamento das classes de manejo serão compensatórios se houver respostas positivas no crescimento e na produção das plantas.

Tabela 4: Modelo ajustado aos semivariogramas, para RP (MPa), estimativas dos dados de efeito pepita (Co), patamar (Co + C), alcance (a), coeficiente de determinação $\left(R^{2}\right)$, Índice de Dependência Espacial e Grau de Dependência Espacial (GDE), em diferentes profundidades do solo $(\mathrm{cm})$

\begin{tabular}{|c|c|c|c|c|c|c|c|c|}
\hline Distância (m) & Camadas (cm) & Modelo & Co & $\mathrm{Co}+\mathrm{C}$ & $\mathbf{a}(\mathbf{m})$ & $\mathbf{R}^{2}$ & IDE & GDE \\
\hline \multirow{4}{*}{0 a 50} & 0 a 10 & Lin & 0,122659 & 0,202504 & 9,75 & 0,110 & 60,6 & Mod \\
\hline & 11 a 20 & Esf & 0,135300 & 0,271600 & 6,70 & 0,601 & 49,8 & Mod \\
\hline & 21 a 40 & Gau & 0,144000 & 0,289000 & 6,62 & 0,760 & 49,8 & Mod \\
\hline & 41 a 60 & Gau & 0,196900 & 0,394800 & 15,40 & 0,480 & 49,9 & Mod \\
\hline \multirow{4}{*}{51 a 100} & 0 a 10 & Gau & 0,034400 & 0,068900 & 2,67 & 0,491 & 49,9 & Mod \\
\hline & 11 a 20 & Lin & 0,089953 & 0,159795 & 9,75 & 0,034 & 56,3 & Mod \\
\hline & 21 a 40 & Lin & 0,113468 & 0,166297 & 9,75 & 0,066 & 68,2 & Mod \\
\hline & 41 a 60 & Lin & 0,082397 & 0,134363 & 9,75 & 0,063 & 61,3 & Mod \\
\hline \multirow{4}{*}{101 a 150} & 0 a 10 & Exp & 0,022600 & 0,067800 & 3,21 & 0,365 & 33,3 & Mod \\
\hline & 11 a 20 & Exp & 0,008200 & 0,060400 & 1,20 & 0,163 & 13,6 & For \\
\hline & 21 a 40 & Lin & 0,0655080 & 0,092736 & 9,75 & 0,059 & 70,6 & Mod \\
\hline & 41 a 60 & Lin & 0,043410 & 0,080010 & 9,74 & 0,013 & 54,3 & Mod \\
\hline \multirow{4}{*}{151 a 200} & 0 a 10 & Lin & 0,091622 & 0,144506 & 9,67 & 0,012 & 63,4 & Mod \\
\hline & 11 a 20 & Lin & 0,064050 & 0,112050 & 9,67 & 0,067 & 57,2 & Mod \\
\hline & 21 a 40 & Lin & 0,076851 & 0,114964 & 9,67 & 0,134 & 66,8 & Mod \\
\hline & 41 a 60 & Lin & 0,120680 & 0,171502 & 9,67 & 0,182 & 70,4 & Mod \\
\hline
\end{tabular}

Lin: linear; Esf: esférico; Exp: exponencial; Gau: gaussiano; Mod: moderado; For: Forte. 


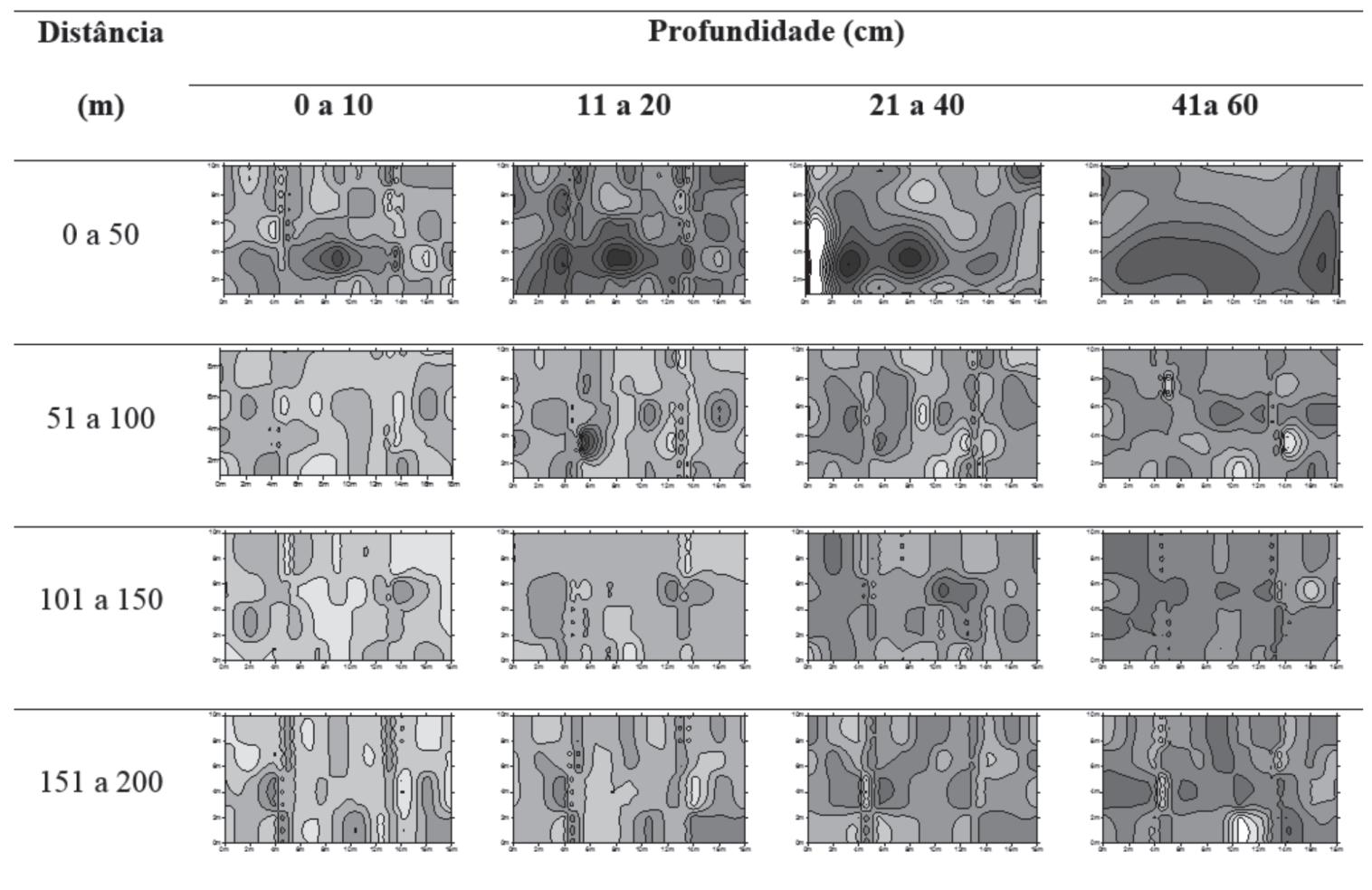

\section{Resistência do solo à penetração (MPa)}

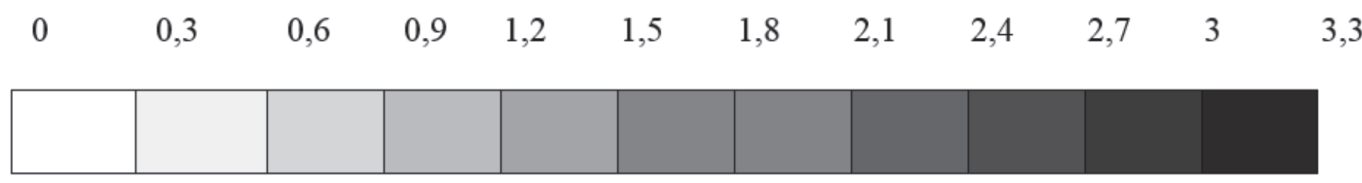

Figura 2: Mapas da distribuição espacial da RP (MPa), nas distâncias e profundidades do solo estudadas.

Tabela 5: Percentil da área estudada dentro de cada classe de RP (MPa), nas diferentes distâncias e profundidades do solo.

\begin{tabular}{|c|c|c|c|c|c|c|c|c|c|c|c|c|}
\hline \multirow[b]{2}{*}{$\mathbf{D}(\mathbf{m})$} & \multirow[b]{2}{*}{$\mathbf{P}(\mathbf{c m})$} & 0,0 & 0,3 & 0,6 & 0,9 & 1,2 & 1,5 & 1,8 & 2,1 & 2,4 & 2,7 & 3,0 \\
\hline & & \multicolumn{11}{|c|}{ \% da área estudada } \\
\hline \multirow{4}{*}{0 a 50} & 0 a 10 & 0,00 & 0,00 & 1,16 & 11,92 & 36,94 & 34,16 & 11,31 & 2,78 & 1,25 & 0,48 & 0,00 \\
\hline & 11 a 20 & 0,00 & 0,00 & 0,12 & 4,22 & 20,63 & 30,53 & 24,80 & 11,76 & 4,53 & 2,27 & 1,14 \\
\hline & 21 a 40 & 0,16 & 12,88 & 50,27 & 25,34 & 10,65 & 0,70 & 0,00 & 0,00 & 0,00 & 0,00 & 0,00 \\
\hline & 41 a 60 & 1,73 & 1,49 & 1,27 & 3,37 & 18,55 & 25,88 & 22,45 & 11,51 & 7,33 & 4,16 & 2,26 \\
\hline \multirow{4}{*}{50 a 100} & 0 a 10 & 0,00 & 0,02 & 7,59 & 45,90 & 41,24 & 5,25 & 0,00 & 0,00 & 0,00 & 0,00 & 0,00 \\
\hline & 11 a 20 & 0,00 & 0,84 & 23,80 & 50,67 & 19,92 & 3,47 & 0,61 & 0,43 & 0,26 & 0,00 & 0,00 \\
\hline & 21 a 40 & 0,00 & 1,44 & 9,37 & 25,41 & 40,16 & 22,35 & 1,27 & 0,00 & 0,00 & 0,00 & 0,00 \\
\hline & 41 a 60 & 0,06 & 1,39 & 2,18 & 8,27 & 53,06 & 30,00 & 5,04 & 0,00 & 0,00 & 0,00 & 0,00 \\
\hline \multirow{4}{*}{100 a 150} & 0 a 10 & 0,00 & 0,00 & 24,34 & 48,80 & 24,54 & 2,32 & 0,00 & 0,00 & 0,00 & 0,00 & 0,00 \\
\hline & 11 a 20 & 0,00 & 1,38 & 17,77 & 66,79 & 13,48 & 0,58 & 0,00 & 0,00 & 0,00 & 0,00 & 0,00 \\
\hline & 21 a 40 & 0,00 & 0,00 & 0,67 & 21,02 & 45,05 & 27,63 & 5,27 & 0,36 & 0,00 & 0,00 & 0,00 \\
\hline & 41 a 60 & 0,00 & 0,00 & 0,68 & 1,66 & 19,54 & 59,79 & 18,32 & 0,01 & 0,00 & 0,00 & 0,00 \\
\hline \multirow{4}{*}{150 a 200} & 0 a 10 & 0,00 & 0,15 & 12,88 & 50,27 & 25,34 & 10,66 & 0,70 & 0,00 & 0,00 & 0,00 & 0,00 \\
\hline & 11 a 20 & 0,00 & 0,00 & 0,70 & 21,95 & 51,20 & 18,77 & 7,38 & 0,00 & 0,00 & 0,00 & 0,00 \\
\hline & 21 a 40 & 0,00 & 0,00 & 0,05 & 4,02 & 24,86 & 41,57 & 28,46 & 1,04 & 0,00 & 0,00 & 0,00 \\
\hline & 41 a 60 & 0,00 & 0,82 & 1,41 & 2,14 & 9,61 & 32,04 & 40,16 & 13,68 & 0,14 & 0,00 & 0,00 \\
\hline
\end{tabular}

D: classes de distância de extração; P: profundidade.

Rev. Ceres, Viçosa, v. 65, n.3, p. 227-233, mai/jun, 2018 


\section{CONCLUSÕES}

O aumento da intensidade de tráfego das máquinas de colheita da madeira no sistema de árvores inteiras contribuiu para as alterações das propriedades físicas do solo.

A área próxima à margem do talhão apresentou maior compactação do solo, causada pela maior intensidade de tráfego do skidder, com valores considerados críticos para o desenvolvimento radicular das plantas.

A técnica de geoestatística mostrou-se viável para a determinação da compactação do solo, nas diferentes regiões do talhão, podendo auxiliar no planejamento das operações de preparo de solo e na redução dos custos de produção.

\section{REFERÊNCIAS}

AguasParaná - Instituto das Águas do Paraná (2017) Totais mensais de chuva. Disponível em: <http://www.aguasparana.pr.gov.br>. Acessado em: 4 de maio de 2018.

Cambardella CA, Moorman TB, Novak JM, Parkin TB, Karlen DL, Turco RF \& Konopka AE (1994) Field scale variability of soil properties in Central Iowa Soils. Soil Science Society America Journal, 58:1501-1511.

Cambi M, Hoshika Y, Mariotti B, Paoletti E, Picchio R, Venanzi R \& Marchi E (2017) Compaction by a forest machine affects soil quality and Quercus robur L. seedling performance in an experimental field. Forest Ecology and Management, 384:406414

Dias Junior MS, Leite FP, Winter ME \& Pires JVG (2003) Avaliação quantitativa da sustentabilidade estrutural de um Latossolo Vermelho-Amarelo cultivado com eucalipto na região de Peçanha-MG. Revista Árvore, 27:343-349.

Embrapa - Empresa Brasileira de Pesquisa Agropecuária (1997) Manual de Métodos de Análise de Solo. 2a ed. Rio de Janeiro, Ministério da Agricultura e do Abastecimento. 212p.

Epron D, Plain C, Ndiaye F, Bonnaud P, Pasquier C \& Ranger J (2016) Effects of compaction by heavy machine traffic on soil fluxes of methane and carbon dioxide in a temperate broadleaved forest. Forest Ecology and Management 382:01-09.

Lapen DR, Topp GC, Gregorich EG \& Curnoe WE (2004) Least limiting water range indicators of soil quality and corn production, Eastern Ontario, Canada. Soil \& Tillage Research, 78:151-170.

Lima JSS, Oliveira PC, Oliveira RB de \& Xavier AC (2008) Métodos geoestatísticos no estudo da resistência do solo à penetração em trilha de tráfego de tratores na colheita de madeira. Revista Árvore, 32:931-938.

Lopes ES, Sampietro JA, Lombardi KC \& Dias AN (2011) Avaliação da umidade na compactação do solo submetido ao tráfego de máquinas de colheita florestal Revista Árvore, 35:659-667.

Machado CC (2014) Colheita florestal. $3^{\mathrm{a}}$ ed. Viçosa, Editora UFV. 543p.

Pavan MA, Bloch MF, Zempulski HC, Miyazawa M \& Zocoler DC (1992) Manual de análise química de solo e controle de qualidade. Londrina, IAPAR. 40p.

Reichert JM, Reinert DJ, Suzuki LEAS \& Horn R (2010) Mecânica do Solo. In: Jong Van Lier Q (Ed.) Física do Solo. Viçosa, Sociedade Brasileira de Ciência do Solo. p.29-102.
Reichert JM, Suzuki LEAS \& Reinert DJ (2007) Compactação do solo em sistemas agropecuários e florestais: Identificação, efeitos, limites críticos e mitigação. In: Cereta CA, Silva LS \& Reichert JM (Eds.) Tópicos em ciência do solo. Viçosa, Sociedade Brasileira de Ciência do Solo. p.49-134.

Seixas F \& Souza CR (2007) Avaliação e efeito da compactação do solo, devido à frequência de tráfego, na produção de madeira de eucalipto. Revista Árvore, 31:1047-1052.

Seixas F, Koury CGG \& Costa LG (2003) Soil compaction and GPS determination of impacted area by skidder traffic. In: $2^{\text {nd }}$ Forest Engineering Conference, Växjö. Proceedings, Skogforsk. p.124-129.

Silva VR, Reichert JM \& Reinert DJ (2004) Variabilidade espacial da resistência do solo à penetração em plantio direto. Ciência Rural, 34:399-406.

Taylor JA, Mcbratney AB \& Whelan BM (2007) Establishing management classes for broadacre agricultural production. Agronomy Journal, 99:1366-1376.

Webster R \& Olivera MA (2007) Geostatistics for environmental scientists. $2^{\mathrm{a}}$ ed. Chichester, John Wiley. 330p.

Zou C, Sands R, Buchan G \& Hudson I (2000) Least limiting water range: A potential indicator of physical quality of forest soils. Australian Journal of Soil Research, 28:947-958. 